\title{
Robust Algorithm for Generalized State Estimation
}

\author{
M. R. Irving
}

\begin{abstract}
This letter introduces a robust generalized state estimator which is able to detect and reject gross measurement errors, parameter errors, and topology errors simultaneously. The solution is based on finding a consistent estimate which minimizes the total number of hypothesized gross errors. The problem is formulated as a mixed integer nonlinear program. A small-scale ac estimation example is given which illustrates some of the properties of the method.
\end{abstract}

Index Terms-Generalized state estimation, mathematical programming, robust estimation.

\section{INTRODUCTION}

G ENERALIZED state estimation algorithms [1]-[3] operate with busbar-level models, so that topology errors (e.g., incorrect circuit-breaker status) can be handled by gross error processing. By extending a previous robust estimator approach [4], [5], a new algorithm is introduced which is capable of simultaneously processing gross measurement errors, gross parameter errors, and topology errors. Test results on a small example network illustrate the capabilities of this method.

\section{PROBLEM Formulation}

In addition to conventional transmission line models, a generalized estimator also models the flow of active and reactive power on "links", where a link represents a zero-impedance connection (such as a closed circuit-breaker). Flows on links and additional voltage magnitudes and phase angles (at their connection points) are included as states of the system. The nonlinear ac loadflow equations then become

$$
\begin{aligned}
& \mathrm{Pi}=\sum \mathrm{Pij}+\sum \mathrm{PLij} \\
& \mathrm{Qi}=\sum \mathrm{Qij}+\sum \mathrm{QLij}
\end{aligned}
$$

where

$$
\begin{array}{ll}
\text { Pi, Qi } & \text { active/reactive injection at bus i; } \\
\text { Pij, Qij } & \text { active/reactive line flow, bus i to j; } \\
\text { PLij, QLij } & \text { active/reactive link flow, bus i to j; } \\
\sum & \text { over connected buses. }
\end{array}
$$

$$
\begin{aligned}
& P i j=f(V i, V j, \theta i, \theta j, \text { Gij, Bij, Bii, Gii }) \\
& \text { Qij }=g(V i, V j, \theta i, \theta j, \text { Gij, Bij, Bii, Gii })
\end{aligned}
$$

where

$$
\begin{array}{ll}
\mathrm{Vi}, \mathrm{Vj} & \text { bus voltage magnitudes; } \\
\theta \mathrm{i}, \theta \mathrm{j} & \text { bus voltage phase angles; } \\
\mathrm{Gij}, \mathrm{Bij} & \text { line conductance and susceptance parameters; }
\end{array}
$$

Manuscript received January 21, 2009; revised March 31, 2009. First published August 25, 2009; current version published October 21, 2009. Paper no. PESL-00007-2009.

The author is with the Brunel Institute of Power Systems, Brunel University, Uxbridge UB8 3PH, U.K.

Digital Object Identifier 10.1109/TPWRS.2009.2030116
Gii, Bii shunt conductance and susceptance at bus i;

$\mathrm{f}, \mathrm{g} \quad$ ac loadflow functions.

Following the principle of adopting an "uncertainty range" for each measured quantity [4], and also introducing uncertainty in the line parameters, we can define a set of uncertainty inequalities:

$$
\mathrm{z}_{\mathrm{k}}-\mathrm{t}_{\mathrm{k}}^{-}-\mathrm{Mb} \mathrm{b}_{\mathrm{k}} \leq \mathrm{z}_{\mathrm{k}}^{\mathrm{e}} \leq \mathrm{z}_{\mathrm{k}}+\mathrm{t}_{\mathrm{k}}^{+}+\mathrm{Mb}_{\mathrm{k}}
$$

where

$\mathrm{Z}_{\mathrm{k}} \quad$ measurement (or parameter) $\mathrm{k}$;

$\mathrm{z}_{\mathrm{k}}^{\mathrm{e}} \quad$ estimated value;

$\mathrm{t}_{\mathrm{k}}^{-} \quad$ lower tolerance;

$\mathrm{t}_{\mathrm{k}}^{+} \quad$ upper tolerance;

$b_{k} \quad 0 / 1$ binary variable;

M arbitrarily large positive scalar value.

The set of uncertainty inequalities include all available measurements of Pi, Qi, Vi, $\theta \mathrm{i}, \mathrm{Pij}$, Qij, PLij, QLij, plus tolerance ranges for $\mathrm{Bij}, \mathrm{Gij}$, Bii, and Gii. The tolerance ranges are defined according to the familiar engineering practice of specifying a quantity as accurate to within plus or minus a tolerance. If all the measurements and parameters are within tolerance, it will be possible to find a solution which satisfies all the above inequalities with $b_{k}=0$. However, if some measurements, or parameters, have unexpectedly large errors, it will be necessary to use $b_{k}=1$ to "switch off" the corresponding uncertainty constraint. The scalar value $\mathrm{M}$ must be chosen to be large in comparison with possible gross errors, in order to eliminate any influence on the solution from "switched off" constraints.

Topology errors can be handled in a similar manner. A link indicated as closed (between bus i and bus $\mathrm{j}$ ) is represented by the following inequalities:

$$
\begin{aligned}
-\mathrm{Mb}_{\mathrm{k}} & \leq \mathrm{Vi}-\mathrm{Vj} \leq \mathrm{Mb}_{\mathrm{k}} \\
-\mathrm{Mb}_{\mathrm{k}} & \leq \theta \mathrm{i}-\theta \mathrm{j} \leq \mathrm{Mb_{ \textrm {k } }} \\
-\mathrm{M}\left(1-\mathrm{b}_{\mathrm{k}}\right) & \leq \mathrm{PLij} \leq \mathrm{M}\left(1-\mathrm{b}_{\mathrm{k}}\right) \\
-\mathrm{M}\left(1-\mathrm{b}_{\mathrm{k}}\right) & \leq \mathrm{QLij} \leq \mathrm{M}\left(1-\mathrm{b}_{\mathrm{k}}\right) .
\end{aligned}
$$

These constraints are "complementary", since the same $b_{k}$ variable is used in both (6) and (7). If the link is actually closed, $b_{k}$ should be zero, constraining the voltages at each end of the link to be equal and switching off the flow constraints. If the link is actually open, $b_{k}$ should be found to be 1 , constraining the link flows to zero and switching off the voltage constraints. A link indicated as open can be represented by similar inequalities, with $\mathrm{b}_{\mathrm{k}}$ and $\left(1-b_{\mathrm{k}}\right)$ interchanged.

The robust estimation criteria used is to select a state estimate that minimizes the total number of measurements, parameters, and link states that are considered out-of-tolerance, i.e.,

$$
\text { Min. } \sum_{\text {all } \mathrm{k}} \mathrm{b}_{\mathrm{k}} \text {. }
$$




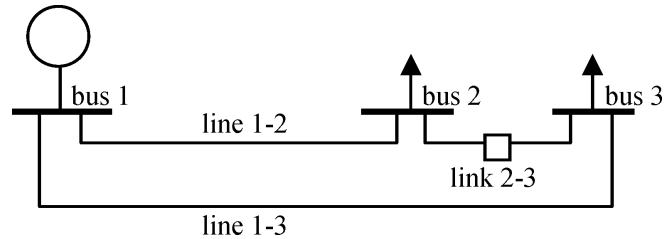

Fig. 1. Example network.

This results in a mixed integer nonlinear program in the binary variables $b_{k}$ and real variables $\mathrm{Vi}, \mathrm{Vj}, \theta \mathrm{i}, \theta \mathrm{j}$, Gij, Bij, Bii, Gii, $\mathrm{PLij}$, and QLij. In the case that a link has no status indication available, inequalities (6) and (7) can be used with the corresponding $b_{k}$ value omitted from the objective function.

\section{RESUlTS AND ANALYSIS}

The problem can be readily solved using standard mathematical programming software, such as the MINLP algorithm of Fletcher and Leyffer, available via the NEOS web-service [6]. The proposed method is therefore related to the branch and bound method of Monticelli [1]. The robustness of the proposed method can be illustrated using the small example network shown in Fig. 1. An accurate loadflow solution was obtained, with the link between bus 2 and bus 3 closed, and with correct network parameters as shown in the second column of Table I. Measurement and parameter uncertainty ranges were then defined, as shown in columns 3 and 4 of Table I. Three simultaneous gross errors were introduced, on $\mathrm{P}_{1}, \mathrm{~B}_{12}$, and the link status (which was erroneously indicated as "open"). The solution of the mixed integer program gave the estimates shown in column 5 of Table I. The three gross errors have been detected and corrected. The minimized cost function has a value of 3 , as the binary variables associated with the gross errors have values of one, with all other binary variables remaining at zero.

A disadvantage of the proposed method is that little noise filtering is provided, as the estimator will use the full range of tolerance available to find an estimate that minimizes the number of hypothesized gross errors. This problem could be counteracted by introducing a second stage of noise filtering using weighted least squares, once the gross errors have been eliminated. Mixed integer nonlinear programming is a computationally demanding task. This limits the online application of the method to small areas of the network where multiple gross errors are suspected and where sufficient local redundancy is available.
TABLE I

UNCERTAINTY RANGES AND ESTIMATES FOR EXAMPLE NETWORK

\begin{tabular}{|l|l|l|l|l|}
\hline & \multicolumn{1}{|c|}{$\mathrm{Z}_{\mathrm{k}}$} & $\mathrm{Z}_{\mathrm{k}}-\mathrm{t}_{\mathrm{k}}{ }^{-}$ & $\mathrm{Z}_{\mathrm{k}}+\mathrm{t}_{\mathrm{k}}{ }^{+}$ & $\mathrm{Z}_{\mathrm{k}}{ }^{\mathrm{e}}$ \\
\hline $\mathrm{P}_{1}$ & 1.824 & 2.81 & 2.84 & 1.822 \\
\hline $\mathrm{Q}_{1}$ & 1.0200 & 1.01 & 1.03 & 1.017 \\
\hline $\mathrm{P}_{12}$ & 1.0423 & 1.03 & 1.05 & 1.031 \\
\hline $\mathrm{Q}_{12}$ & 0.5828 & 0.575 & 0.59 & 0.575 \\
\hline $\mathrm{P}_{31}$ & -0.7714 & -0.78 & -0.763 & -0.78 \\
\hline $\mathrm{Q}_{31}$ & -0.3857 & -0.388 & -0.381 & -0.388 \\
\hline $\mathrm{P}_{2}$ & -0.9 & -0.91 & -0.89 & -0.906 \\
\hline $\mathrm{Q}_{2}$ & -0.45 & -0.455 & -0.445 & -0.445 \\
\hline $\mathrm{P}_{3}$ & -0.9 & -0.91 & -0.89 & -0.89 \\
\hline $\mathrm{Q}_{3}$ & -0.45 & -0.455 & -0.445 & -0.447 \\
\hline $\mathrm{V}_{1}$ & 1.0 & 0.99 & 1.01 & 0.99 \\
\hline $\mathrm{V}_{2}$ & 0.9630 & 0.95 & 0.97 & 0.952 \\
\hline $\mathrm{G}_{12}$ & 4.0 & 3.96 & 4.04 & 3.96 \\
\hline $\mathrm{B}_{12}$ & -20.0 & -10.1 & -9.9 & -19.33 \\
\hline $\mathrm{G}_{13}$ & 3.0 & 2.97 & 3.03 & 3.03 \\
\hline $\mathrm{B}_{13}$ & -15.0 & -15.15 & -14.85 & -14.85 \\
\hline Link $_{23}$ & closed & open & open & closed \\
\hline
\end{tabular}

\section{CONCLUSIONS}

A robust generalized state estimator has been presented. The use of binary decision variables throughout (to accept or reject measurements, parameter values, and topological states) allows all three types of gross errors to be judged on a comparable basis. Simultaneous gross errors in measurements, parameters, and topology can be identified and corrected. The computational effort of solving the required mathematical program probably limits the practical application of the method to relatively small networks, or sections of the network, at present. However, it is hoped that future advances in numerical algorithms and computer hardware will widen the scope of application in the future.

\section{REFERENCES}

[1] A. Monticelli, State Estimation in Electric Power Systems-A Generalized Approach. Norwell, MA: Kluwer, 1999.

[2] H. Singh and F. L. Alvarado, "Network topology determination using least absolute value state estimation," IEEE Trans. Power Syst., vol. 10, no. 3, pp. 1159-1165, Aug. 1995.

[3] E. M. Lourenco, A. S. Costa, and K. A. Clements, "Bayesian-based hypothesis testing for topology error identification in generalized state estimation," IEEE Trans. Power Syst., vol. 19, no. 2, pp. 1206-1215, May 2004

[4] M. R. Irving, "Robust state estimation using mixed integer programming," IEEE Trans. Power Syst., vol. 23, no. 3, pp. 1519-1520, Aug. 2008 .

[5] S. Gastoni, G. Granelli, and M. Montagna, "Robust state estimation procedure based on the maximum agreement between measurements,' IEEE Trans. Power Syst., vol. 19, no. 4, pp. 2038-2043, Nov. 2004.

[6] NEOS, NEOS Server for Optimisation, 2008. [Online]. Available: http://neos.mcs.anl.gov/neos. 